\title{
VISUAL EXPERIMENT \\ Estimation of aflatoxins in peanut or maize by enzyme linked immunosorbent assay
}

\author{
Shruti Shukla \\ Department of Food Science and Technology, Yeungnam University, Gyeongsan-Si, Gyeongsanbuk-Do \\ 712-749, Republic of Korea.
}

Correspondence: shruti.shukla15@yahoo.com; Fax: +82-53-810-4662

Published on: 16 June, 2016

DOI: $10.3329 /$ bjp.v11i3.28178

\section{ABSTRACT}

Aflatoxin are potentially toxic metabolites produced by microscopic fungi when grow on food. Hence, determining content of mycotoxins such as aflatoxin has significant pharmaceutical value. This study reports step-by-step visual demonstration of determination of aflatoxin contents in peanut and maize (corn)-based food samples through employing enzyme linked immunosorbent assay (ELISA). The method is based on direct competitive ELISA in a micro-well format which allows determination of exact amount of aflatoxin in food samples as parts per billion (ppb), and the results are calculated using Neogen's Veratox data reduction software that converts the absorbance values into quantities. This visual experiment will certainly contribute knowledge regarding importance and applicability of the ELISA method for determining aflatoxin content in variety of food samples avoided of analytical issues such as sampling, laborious sample preparation, and critical selection of analytical methods.

\section{INTRODUCTION}

Mycotoxins are secondary metabolites of microscopic fungi which are not indispensable to the fungi's life but show toxic effects on human beings, animals, plants and microorganisms. The toxin-producing fungi include Penicillium, Aspergillus and Fusarium being developed on agricultural food products including corn, wheat, rice, maize (corn), spices, dried fruits, and nuts (Laszczynska et al., 2001). Especially several species of genera Aspergillus such as A. flavus and A. parasiticus produce various types of highly toxic aflatoxins such as B1, B2, G1 and G2. These fungi can grow on a wide variety of foods and feeds under favorable temperature and humidity. Contamination by aflatoxins can take place at any point along the food chain from the field, harvest, handling, shipment and storage (Giray et al., 2007). In addition, aflatoxins have been clearly identified as highly toxic, mutagenic, teratogenic and carcinogenic compounds and have been implicated as causative agents in human hepatic and extrahepatic carcinogenesis (Castells et al., 2008). Aflatoxin B1 (AFB1) is most frequently found in plant substrates, which has shown the highest toxigenic potential (Moss, 1998). Based on previous studies, the IARC (1993) has classified AFB1 as a class 1A human carcinogen (Rothschild, 1992). In 2002, Brazilian authorities established a maximum limit of $20 \mu \mathrm{g} / \mathrm{kg}$ for total aflatoxins (B1 + B2 + G1 + G2) in peanuts (unshelled, shelled, raw or roasted), peanut paste (or peanut butter) and corn (whole, crushed, mashed, ground, meals and brans) (Hoeltz et al., 2012).

There are several analytical and conventional methods available for the determination of aflatoxins in a variety of samples (food and feed, pharmaceutical medicines, vegetables, nuts, etc). However, immunochemical methods with the use of antigen-antibody reactions belong to selective, sensitive and fast techniques, being relatively inexpensive at the same time. Recently, they are more frequently used for the determination of food components (Leszczynska et al., 2001; Mohammad Reza et al., 2012; Leszczynska et al., 2001).

Research on food products has demonstrated that total aflatoxins are still being found frequently in food product at level that are of significant concern for consumers (Stroka et al., 2002). Therefore, in the present 
visual experiment, we aimed to demonstrate the fast and reliable protocol for researchers to determine aflatoxin level in peanut, and/or maize-based commercial food products by using Veratox total aflatoxin detection kit which is based on competitive direct enzyme-linked immunosorbent assay (CD-ELISA).

\section{MATERIALS AND EQIPMENTS}

\section{Major requirements}

Veratox total aflatoxin text kit (Neogen corp.)

96-microtiter plate well holder

Distilled water

Test samples

\section{Disposables}

Pipette tips for micropipettor

Eppendorf tubes $(1.5 \mathrm{~mL})$

Syringe filters $(0.45 \mu \mathrm{m})$

96-well microtiter plate

$15 \mathrm{~mL}$ falcon tubes

Paper towels

\section{Equipments}

Spectrophotometer (Tecan)

Vortex

Multichannel pipette

\section{VIDEO CLIPS}

Test sample preparation: $7 \mathrm{~min} 12 \mathrm{sec}$

Assay procedure: $5 \mathrm{~min} 23 \mathrm{sec}$

Measurement of absorbance by Tecan ELISA microtiter plate reader: 2 min $57 \mathrm{sec}$

\section{METHOD}

\section{Preparations}

1. Prepare a $70 \%$ methanol solution by using anhydrous grade methanol

2. Blend $5 \mathrm{~g}$ of sample with $25 \mathrm{~mL}$ of $70 \%$ methanol for $1 \mathrm{~min}$ in a high speed mixer

3. Transfer the homogenized samples to the stomach bag and allow for further 3 min shaking

4. Incubated the samples under the clean bench at room temperature for 4-5 hours

5. Filter the extract by using vacuum filtration device

6. Re-filter the extract into Eppendorf tubes through syringe filter $(0.45 \mu \mathrm{m})$

7. The sample is now ready for testing

[Extraction procedure for the same sample should be done in triplicate in order to avoid any sampling error during analysis]

Assay procedure

1. Add $100 \mu \mathrm{L}$ conjugate to red marked mixing wells 
2. Add $100 \mu \mathrm{L}$ controls and samples to the red marked mixing wells

3. Using multichannel pipettor, mix the liquid in the wells by pipetting it up and down at least 3 times

4. Transfer $100 \mu \mathrm{L}$ sample to antibody wells and incubate for $2 \mathrm{~min}$

5. Mix the sample in the wells for first $10-20 \mathrm{sec}$ of incubation by sliding the well holder

6. Dump liquid from antibody wells on to paper towel

7. Wash the wells thoroughly 5 times with deionized water

8. Tap out water on absorbent paper towel

9. Transfer $100 \mu \mathrm{L}$ substrate from reagent boat to antibody wells using 12 -channel pipettor, andincubate for $3 \mathrm{~min}$

10. Transfer $100 \mu \mathrm{L}$ red stop solution from reagent boat to antibody wells

11. Read results using a micro-plate reader with a $650 \mathrm{~nm}$ filter

[Wipe the bottom of well plate with tissue paper towel]

[Air bubble should be eliminated, they might effect to analytical results.]

[Tested samples should be analyzed in triplicates in different sets in order to avoid errors]

\section{DISCUSSION}

The results for the quantitative analysis of aflatoxin levels, determined in tested peanut and maize (corn) food product samples by veratox total aflatoxin test kit (enzyme immunoassay), were in the range of 2.2$11.2 \mu \mathrm{g} / \mathrm{kg}$. The assay was based on direct competitive ELISA where the free aflatoxin in the samples and controls is allowed to compete with enzyme labeled aflatoxin (conjugate) for the antibody binding sites. After a wash step, substrate is added, which reacts with the bound conjugate to produce the blue color. More blue color means less aflatoxin. The test is read in a micro-plate reader to yield optical densities. The optical densities of the controls from the standard curve, and the sample optical densities are plotted against the curve to calculate the exact concentration of aflatoxin. The ELISA method showed a maximum limit of $11.2 \mu \mathrm{g} / \mathrm{kg}$ for aflatoxin levels in the tested samples of peanut sample, which was under the safety limit allowed by the Codex Alimentarius Commission (CAC) regulatory agency (CAC, 2001).

Aflatoxin is considered to be the most potent, naturally-occurring carcinogen which has been linked to a variety of health problems in both humans and animals. When aflatoxin contaminated feed is eaten, it can cause various diseases such as liver damage, cancer, mental impairment, edema, and hemorrhaging etc (Castells et al., 2008). The United States Food and Drug Administration (USFDA) has set a maximum allowable level of total aflatoxins at 20 parts per billion (ppb). However, the Codex Alimentarius Commission (CAC), a joint FAO/WHO Food Standards Program, adopted a hazardous limit of $15 \mu \mathrm{g} / \mathrm{kg}$ for total aflatoxin (CAC, 2001). Commodities used for human and animal consumption must be tested to ensure that aflatoxin levels are below this number.

\section{REFERENCES}

Castells M, Marin S, Scanchis V, Ramos AJ. Distribution of fumonisins and aflatoxins in corn fractions during industrial cornflake processing. Int J Food Microbiol. 2008; 123: 81-87.

Codex Alimentarius Commission (CAC) Joint FAO/WHO food standards programme, codex committee on food additives and contaminants. Thirty third session of CODEX. Hague, Netherland. 2001.

Giray B, Girgin G, Engin AB, Aydin S, Sahin G. Aflatoxin levels in wheat samples consumed in some regions of Turkey. Food Control. 2007; 18: 23-29.

Hoeltz M, Einloft TC, Oldoni VP, Dottori HA, Noll IB. The occurrence of aflatoxin B1 contamination in peanuts and peanut products marketed in Southern Brazil. Braz Arch Biol Technol. 2012; 55: 313-17.

Leszczynska J, Maslowska J, Owczarek A, Kucharska U. Determination of aflatoxins in food products by the ELISA method. Czech J Food Sci. 2001; 19: 8-12.

Mohammad Reza SS, Masoud A, Ali T, Faranak G, Mahboob N. Determination of aflatoxins in nuts of Tabriz confectionaries by ELISA and HPLC methods. Adv Pharm Bull. 2012; 2: 123-26. 
Moss M.O. Recent studies of mycotoxins. J Appl Microbiol. Symposium Suppl. 1998; 84: 62S-76S.

Rothschild LJ. IARC classes AFB1 as class 1 human carcinogen. Food Chem. 1992; 34: 62-66.

Stroka J, Anklam E. New strategies for the screening and determination of aflatoxins and the detection of aflatoxin-producing moulds in food and feed. Trends Anal Chem. 2002; 21: 90-95.

\section{PRECAUTIONS}

Store test kit refrigerated, between $2-8^{\circ} \mathrm{C}$ when not in use. Do not freeze.

Kits should be brought to room temperature prior to use.

Keep wells sealed in the foil pouch until needed. Remove wells from the foil pouch only after samples are extracted, and the test is set to begin.

Avoid prolonged storage of kits at ambient temperatures.

Follow proper pipetting techniques, including priming of tips. 


\section{Your feedback about this paper}

1. Number of times you have read this paper 0

2. Number of times you have seen the video clip 0

3. Quality of paper Click

4. Your comments

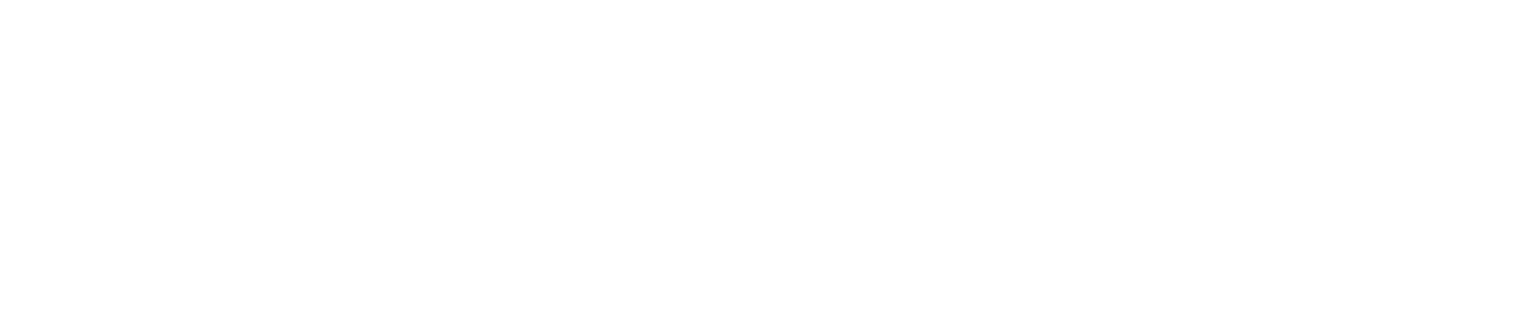

